\title{
SISTEMA DE INTERACCIÓN VISUAL PARA UN ROBOT SOCIAL
}

\author{
Mario Domínguez López \\ Universidad de Valladolid, Escuela de Ingenierías Industriales. \\ Paseo del Cauce 59, 47011 Valladolid \\ ikermario@gmail.com \\ Samuel Marcos Pablos \\ Centro Tecnológico CARTIF. Parque Tecnológico de Boecillo, 205, 47151 Boecillo, Valladolid \\ sammar@cartif.es \\ Eduardo Zalama Casanova, Jaime Gómez García-Bermejo \\ Universidad de Valladolid, Escuela de Ingenierías Industriales, ITAP-DISA. \\ Paseo del Cauce 59, 47011 Valladolid \\ ezalama@eii.uva.es,jaigom@eii.uva.es
}

\section{Resumen}

Una de las características de los robots sociales es que tengan expresividad y fluidez lo más cercana a la naturaleza humana. En este caso una de las mayores fuentes de expresividad son los rasgos faciales. Por otra parte, estamos acostumbrados a comunicarnos mirando a otras personas a la cara por lo que desarrollar robots con expresividad, capacidad gestual y de seguimiento facial es una de las características buscadas en el desarrollo de robots sociales.

El presente artículo describe el desarrollo de un sistema de interacción hombre-máquina para un robot móvil social constituido por dos subsistemas o módulos. El primer subsistema se encarga de la detección del interlocutor y su seguimiento facial. Para ello realiza una detección inicial a partir de la información suministrada por el láser de navegación del robot, para posteriormente fijar la atención a partir de la información obtenida con una cámara de rango tipo Kinect. El segundo subsistema de encarga de la generación de gestos del robot. Para ello se ha adoptado el estándar FACS (Facial Action Coding System) lo cual permite su utilización en diferentes tipos de robots. La generación de expresiones se realiza mediante la combinación del movimiento controlado de cuello, párpados y ojos, con gestos mostrados en un display luminoso en la boca del robot.

Palabras clave: FACS, ROS, Interacción hombremáquina, Robot social.

\section{INTRODUCCIÓN}

Un robot social es aquel que comunica e interactúa con las personas de forma sencilla y agradable siguiendo comportamientos, patrones y normas sociales. Para ello, además de tener apariencia humanoide, se necesita que disponga de habilidades que se enmarcan dentro del dominio de la llamada inteligencia social. Se debe tener en cuenta que la socialización con las personas es un tema difícil, ya que los robots y los humanos no comparten un lenguaje común ni perciben el mundo de la misma forma [12].

Según Dauthenhahn [3] los robots sociales son agentes personalizados que forman parte de un grupo heterogéneo. Para llevar a cabo estos objetivos un robot tiene que ser capaz de mostrar ciertas características: personificación, emoción, diálogo, personalidad, percepción orientada a humanos, modelado del entorno, aprendizaje social, e intencionalidad. Se considera muy importante que posean una interfaz natural con algún grado de representación antropomórfica tal como una cabeza (personificación), con la que pueda realizar expresiones faciales para comunicar estados de ánimo de manera gestual (emociones).

Por ello en los últimos años los investigadores han tratado de desarrollar robots con personificación, capacidad gestual y sistemas de percepción y control que faciliten la interacción con las personas.

La entidad corpórea y capacidad gestual de los robots facilita la interacción, al fin y al cabo desde siempre nos hemos comunicado con otras personas cara a cara. El aspecto del robot también ha sido discutido ampliamente desde que M. Mori [8] definió la regla 
básica sobre la familiaridad y empatía en función de su grado de similaridad a las personas. Sin embargo, es necesario de dotar a los robots de capacidades de interacción inspiradas en las de los humanos.

En este artículo se presenta el desarrollo de un sistema de seguimiento facial y de generación gestual para Sacarino, un robot desarrollado en Fundación Cartif. El artículo está organizado de la siguiente forma. En la sección 2 se describe Sacarino, el robot en el que se han implementado los sistemas de interacción descritos en este artículo. En la sección 3 se explica el sistema de seguimiento Facial. En la sección 4 se explica el sistema de generación gestual. En la sección 5 se aborda el sistema global de interacción para finalizar con las conclusiones en la sección 6 .

\section{EL ROBOT SACARINO II}

En investigaciones precedentes nuestro grupo de investigación ha desarrollado el robot social Sacarino, integrado por una base móvil y un sistema de interacción antropomorfo $[8,11]$. El robot ha sido probado de forma intensiva en un entorno hotelero, durante largos periodos de tiempo.

Fruto de esta experimentación se ha abordado el desarrollo de un nuevo robot, Sacarino II (Figura 2). Este robot tiene como finalidad ofrecer servicio en un hogar con personas mayores, donde desempeñará funciones de acompañamiento, juegos, actividades físicas, información, apoyo a terapia etc. El nuevo robot es más ligero y estilizado que la versión precedente, tiene tracción diferencial y en él se ha integrado una Tablet Android que permite utilizar multitud de aplicaciones y servicios de Google Play, en particular el servicio de reconocimiento de voz de Google. Además, este elemento sirve para apoyar la comunicación oral cuando el reconocimiento de voz y la gestión de diálogo se ven comprometidos, especialmente en ambientes ruidosos y poco estructurados.

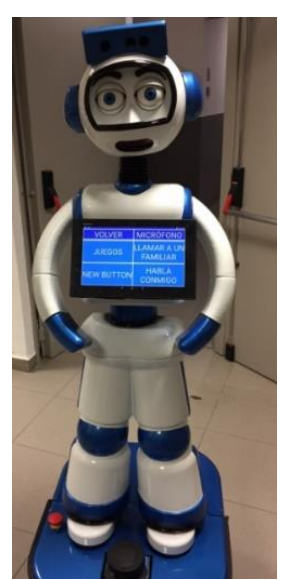

Figura 2. Robot Sacarino II
La Figura 3 se muestra la estructura básica de la cabeza del robot, que es su elemento fundamental de interacción. Además, para facilitar la percepción del entorno y de los usuarios se ha incluido un sensor Kinect en la parte superior de dicha cabeza, que se complementa con el láser de navegación situado en la base del robot.

El presente trabajo se centra en dos aspectos específicos de la interacción usuario-robot: que la cabeza siga al interlocutor con la mirada y que sea capaz de generar un conjunto de expresiones emocionales básicas.

A partir de una primera detección del usuario mediante un sensor de visión $2 \mathrm{D}$ como es el láser SICK, el robot en modo de funcionamiento de seguimiento, pasará a realizar un seguimiento facial del usuario mediante Microsoft Kinect. La principal novedad aquí es el diseño de una función de filtrado tal, que consigue transiciones suaves y fluidas en el movimiento facial, lo que provoca un impacto positivo sobre la interacción del robot con las personas.

Por su parte, en la generación de emociones resulta novedosa la adopción del estándar FACS (Facial Action Coding System), que permite la formulación generalista de expresiones y gestos faciales, reforzando la interacción usuario-robot.

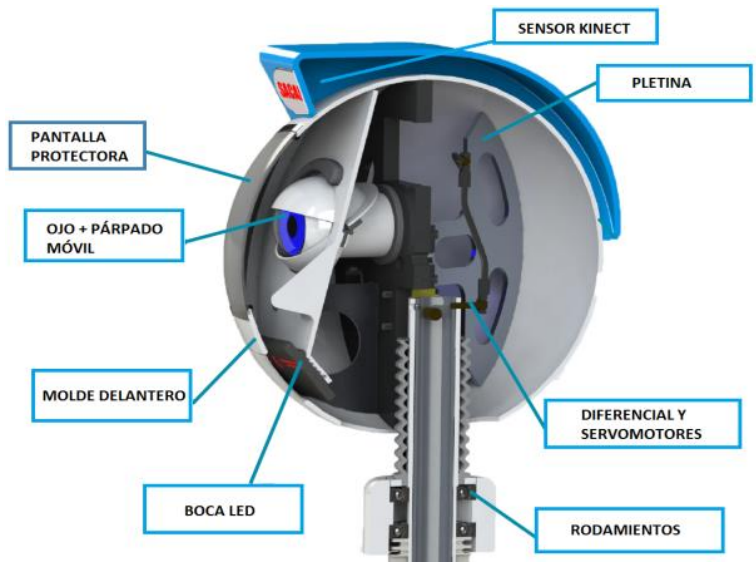

Figura 3: Detalle de la cabeza de Sacarino II

La cabeza integra seis servomotores controlados por el driver SCS32. Tres de estos motores se encargan de gestionar los grados de libertad del cuello y el resto se destinan al movimiento de párpados y cejas. Además, Sacarino II cuenta con una pantalla de matriz de leds como boca, que le permite acompañar los diferentes gestos de cabeza, cejas y párpados con muecas labiales, dotando a así a la cara de una mayor expresividad.

Tanto el sistema de seguimiento facial como el de generación de gestos se han desarrollado sobre Robot Operating System, ROS [7]. Se trata de un sistema 
operativo de código abiero (Open source) que cuenta con una comunidad desarrolladora muy extensa y facilita la programación modular basada en componentes, y abstracción hardware.

\section{MÓdULO DE SEGUIMIENTO FACIAL}

Para establecer una interacción satisfactoria el robot tiene que cumplir una serie de normas básicas de actuación cuando se encuentra ante un humano. En concreto, una de las reglas básicas de comportamiento es mirar a la persona que se tiene delante. El presente apartado aborda este aspecto.

\subsection{OBTENCIÓN E INTERPRETACIÓN DE DATOS}

Como elementos sensores para realizar el seguimiento del usuario se ha optado por combinar el sensor Microsoft Kinect situado en la cabeza del robot, con el láser SICK situado en la base móvil. Este último proyecta un haz de luz en un arco de $270^{\circ}$ y es utilizado también durante la navegación del robot por el entorno.

Para tratar los datos que ofrece Kinect se han seleccionado las librerías OpenNi, disponibles en ROS, que procesan la información suministrada por el sensor de Kinect a formatos que pueden ser utilizados para el trabajo a alto nivel.

En concreto, la librería OpenNi_Tracker [10] es un nodo ROS que, mediante técnicas de tratamiento de imagen (segmentación y esqueletización) en conjunto con la nube de puntos, es capaz de situar sistemas de referencia en las articulaciones de un usuario que se encuentre dentro del campo visual de Kinect. Esto se logra a partir de una serie de matrices de transformación, denominadas Transformed Frames.

Por su parte, el láser de la base se utiliza en la detección de las piernas de los usuarios. En primer lugar, un nodo ROS llamado leg_detector [9], se encarga de, mediante un algoritmo probabilista, encontrar posibles conjuntos de puntos (clusters) que puedan tener la morfología de piernas humanas. Seguidamente, refiere cada uno de los puntos a un sistema de referencia situado en el propio láser mediante los ya comentados Transformed Frames.

Finalmente, este nodo ofrece como salida un vector de posibles personas detectadas.

\subsection{ALGORITMO DE SEGUIMIENTO CON KINECT}

Para lograr el objetivo de que el robot dirija la mirada hacia el usuario, los ángulos (panorámico y cabeceo) que forman la orientación de referencia de la cabeza del usuario, respecto del de Kinect, deben ser llevados a 0 por el sistema de control.

Para el control de movimiento de la cabeza y resto de elementos (párpados y cejas) se utiliza un conjunto de servomotores y la tarjeta controladora SSC32 de Lynxmotion [13], capaz de controlar hasta 32 servomotores mediante un microcontrolador Atmel Atmega168 de $14.74 \mathrm{MHz}$.

Uno de los problemas importantes a la hora de hacer seguimiento, es que los movimientos de la cabeza sean suaves evitando oscilaciones al alcanzar la posición de referencia para lo que se ha introducido un controlador no lineal con zona muerta y saturación mediante una expresión sigmoidal. La expresión viene dada por:

$$
f(e)=\operatorname{sgn}(e) \cdot \frac{e_{\max }}{1+e^{-\left(2 e-e_{\max }\right)}}
$$

donde $e$ es el error, $\operatorname{sgn}(e)$ es la función signo que da un valor +1 o -1 dependiendo de si el error es mayor o menor que cero y $e_{\max }$ es el error máximo admisible.

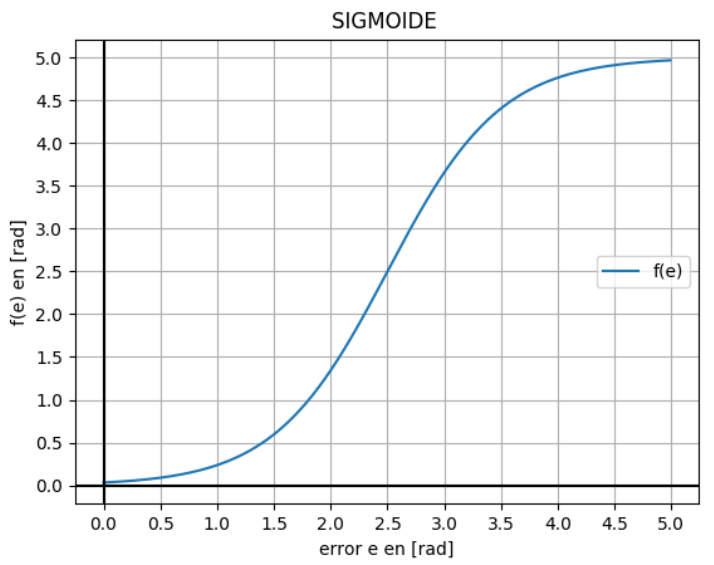

Figura 5. Función sigmoide definida en ecuación (1).

La sigmoide limita la acción de control para valores grandes del error y la limita ante errores pequeños. Esto último permite restringuir el movimiento ante pequeños desplazamientos del usuario, evitando oscilaciones.

La referencia de velocidad que se envía a la tarjeta controladora es la máxima admitida para cada servomotor pues la tarjeta controladora se encarga de generar los perfiles de aceleración y deceleración.

\subsection{ESTRATEGIA DE SEGUIMIENTO CON EL LÁSER SICK}

El láser SICK se utiliza para obtener la posición de un usuario en función de la detección de pares de 
piernas, para, tras esto, hacer un seguimiento del usuario con la cabeza del robot, restringido al plano panorámico o de guiñada (primer grado de libertad).

Como se introducía en (3.1), la salida tras los nodos de tratamiento e identificación de pares de piernas devuelve un vector de posibles personas detectadas (punto medio entre dos piernas candidatas). Cada uno de los objetos persona en el vector, tiene un campo llamado fiabilidad (reliability), que se mueve en un intervalo de 0 a 1 y da muestra de cuán seguro es que verdaderamente se trate de una persona real (no de ruido $\mathrm{u}$ otros objetos).

La estrategia seguida comienza por ordenar este vector de mayor a menor fiabilidad, escogiendo como valor inicial el primer elemento.

A continuación, se compara un umbral para evitar hacer seguimiento a elementos ruidosos, con perturbaciones u objetos. Para ello se ha fijado un umbral de $0.2(20 \%)$.

A partir de este momento, el sistema de control toma nota del número de identificador de esa persona, $\mathrm{y}$, en cada iteración la busca por dicho identificador, con el fin de realizar un seguimiento estable de la misma incluso en presencia de otras personas.

Las únicas dos condiciones que se pueden dar para que el robot cambie de persona a la que hace el seguimiento son las siguientes:

1. Que la persona salga del rango de alcance del láser, en cuyo caso, volveremos a tomar del vector de entrada, la persona que tenga la fiabilidad más alta.

2. Que la persona seguida actualmente permanezca estática y que, a su vez, otra persona varíe su posición de manera significativa, en cuyo caso se pasará a seguir a esta última.

Con objeto de enviar la referencia angular a la controladora $\mathrm{y}$, por tanto, al servomotor de movimiento panorámico del cuello, se calcula el ángulo que forman el punto medio del par de piernas y el sistema de referencia láser mediante:

$$
\theta=\arctan \frac{y}{x}
$$

donde $x$ e $y$ corresponden al punto medio del par de piernas seleccionado.

\section{MÓDULO DE EXPRESIÓN GESTUAL}

Otro de los ingredientes que se ponen en juego en toda interacción humana son todas aquellas acciones que tienen que ver con la comunicación no verbal, es decir, con el complejo sistema de gestos.

El saber interpretar qué movimientos dan lugar a ciertas emociones no es tarea fácil. Se trata de un campo de estudio bastante amplio y que comprende varias disciplinas, no solamente técnicas, sino que incumben a la medicina o psicología, por ejemplo.

De hecho, el primero en formular el problema en forma de clasificación fue el psicólogo Paul Ekman en los años 70 [6]. Propuso un estándar muy utilizado en la actualidad conocido como estándar FACS (Facial Action Coding System) que asigna una determinada emoción a un conjunto concreto de músculos faciales, cada uno activado a un determinado nivel de intensidad.

La unidad básica del sistema FACS es la unidad de acción, o Action Unit (UA). Una UA representa las actividades musculares que producen cambios momentáneos en las apariencias faciales. Dicho de otra manera, una unidad de acción es un código numérico para describir los movimientos de los músculos faciales.

Tabla 1: Codificación de unidades de acción en Sacarino II basada en FACS

\begin{tabular}{|c|c|}
\hline Identificador & Descripción \\
\hline 2 & Levantamiento exterior de cejas. \\
\hline 3 & Bajar cejas. \\
\hline 45 & Bajar párpados. \\
\hline M45 & Levantamiento de párpados. \\
\hline 46 & Guiño ojo izquierdo. \\
\hline M46 & Guiño ojo derecho. \\
\hline 47 & Apertura de ojos. \\
\hline 51 & Movimiento cuello izquierda. \\
\hline 52 & Movimiento cuello derecha. \\
\hline 53 & Alzar cuello. \\
\hline 54 & Bajar cuello. \\
\hline 57 & Inclinar cabeza izquierda \\
\hline 58 & Inclinar cabeza derecha \\
\hline
\end{tabular}

La relación entre las $\mathrm{AU}$ y los músculos faciales no es necesariamente 1-a-1; algunas UA se componen de más de un músculo y otras UA se basan en describir movimientos separados del mismo músculo.

El acto de describir movimientos faciales utilizando el sistema de codificación facial se llama FACS de codificación. Códigos múltiples FACS se notan usando más ('+'). Por ejemplo $1+2$ representa elevar las partes interior y exterior de la ceja (es decir, elevar toda la ceja).

El FACS también incluye un sistema para describir la intensidad de cada AU mediante la colocación de una letra de $\mathrm{A}$ a $\mathrm{E}$ después del código numérico, pero 
antes del signo más. El rango de intensidad para cada letra es:

- $\quad$ Rastro (A)

- Leve (B)

- Marcado / Pronunciado (C)

- $\quad$ Severo (D)

- Extremo / Máximo (E)

Para continuar con el ejemplo anterior, $1 \mathrm{E}+2 \mathrm{E}$ representa la elevación extrema (o máxima) de las porciones interior y exterior de las cejas.

Pongamos un caso real que se puede dar en el robot, sea el gesto "sorpresa" el que tiene que expresar Sacarino II. Las unidades de acción que se deben activar serían la 1 con intensidad E (Levantar las cejas al máximo), levantamiento labio superior (unidad de acción 10) a intensidad severa D, levantar párpado superior 5 también con intensidad D y por último alzar la cabeza (53) con intensidad marcada, C. Es decir:

$$
\text { Sorpresa }=1 E+10 D+5 D+53 C
$$

En Sacarino II se han implementado las unidades de acción expuestas en la Tabla 1 y cuya combinación da lugar a las seis emociones básicas además de la expresión neutra, que son: tristeza, asco, miedo, alegría, enfado, sorpresa.

\subsection{IMPLANTACIÓN}

Para llevar a cabo este subsistema de expresiones en el robot, se ha optado por una estructura modular, que aprovecha el potencial de ROS en este aspecto, tal como ya ha sido comentado. Esto significa que se ha seguido una programación jerárquica en la que cada nivel inferior proporciona una serie de servicios al nivel superior, y este no se debe de preocupar de cómo lo lleva a cabo.

\subsubsection{Servidor de acciones}

Cada gesto es una combinación de unidades de acción y, a su vez, cada unidad de acción se concreta en el movimiento de ciertos servomotores.

Este nodo ROS se encarga de recibir como entrada las unidades de acción que le llegan del cliente (nivel 2) y traducirlas en movimiento de servomotores, lo cual significa dar el identificador del servomotor, y su posición y velocidad. Una vez realizado este cálculo, publica un vector con la cadena de servomotores, especificando para cada uno posición y velocidad, al servicio de la controladora para que esta mueva los servomotores.

\subsubsection{Cliente de acciones}

Se encuentra en el nivel 2 de la arquitectura. Siguiendo un paradigma cliente-servidor este nodo se comunica con el servidor de acciones.

Recibe como entrada la expresión que se desea realizar, para, seguidamente, hacer la correspondencia entre el gesto y las unidades de acción, intensidad y velocidad que le corresponden. Una vez termina, ofrece como salida un vector con la estructura expuesta en la ecuación (3) y que sirve como entrada del servidor de acciones.

La arquitectura de los subsistemas de seguimiento y de expresión gestual se muestran en la Figura 6.

A continuación, se hablará de la última capa en la arquitectura que sincroniza ambos módulos según ordene la entidad que gobierna el robot entero, el secuenciador.

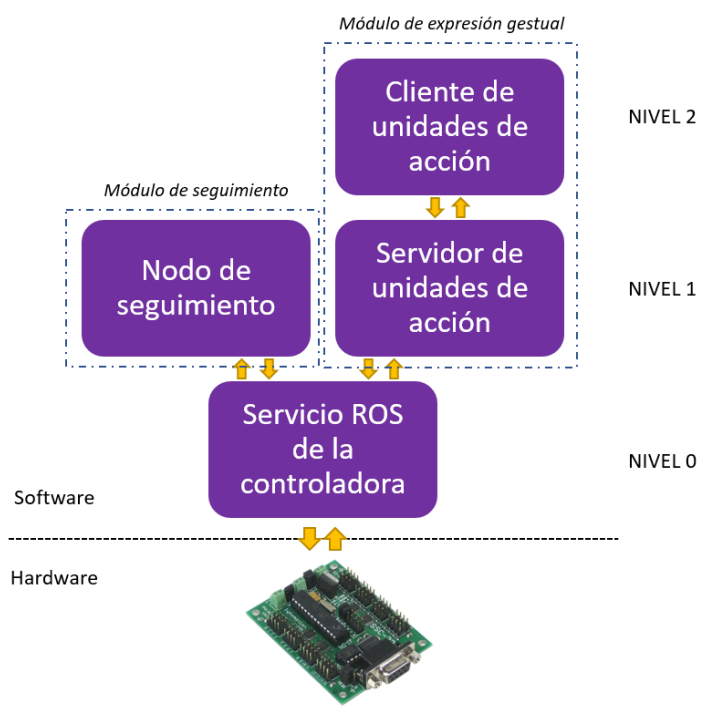

Figura 6: Arquitectura de los subsistemas

\section{SISTEMA GLOBAL DE INTERACCIÓN}

Con objeto de unificar los subsistemas vistos hasta ahora se ha aprovechado una herramienta potente incluida en ROS, como es la librería de acciones ("actionlib").

En definitiva, lo que se persigue es una última capa superior en la arquitectura que sirva de interfaz entre todo el sistema de interacción y el secuenciador del robot. La librería de acciones parte del paradigma cliente-servidor pero incluyendo nuevos matices.

Básicamente, este nuevo modelo de comunicación permite que el cliente reciba información del estado de su petición en tiempo real ("feedback"), en lo que 
espera el resultado final del servidor. Además, ahora es posible, por parte del cliente, cancelar las peticiones que hace al servidor en tiempo real.

Hemos definido una serie de comandos de alto nivel, que van a ir siendo modificados por el secuenciador:

- Modo de seguimiento

- Modo interacción

- Modo descanso

De esta manera, el secuenciador de programa irá ordenando el cambio de modo de acuerdo a estímulos externos.

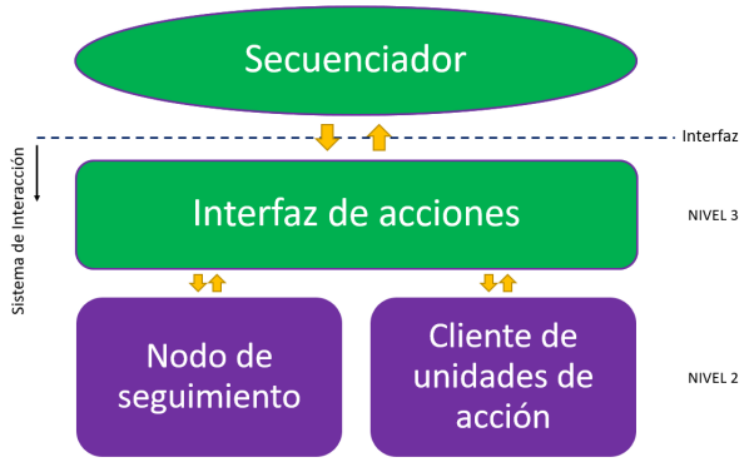

Figura 7: Nivel superior de la arquitectura

Cuando el robot se encuentre en modo seguimiento, solamente se centrará en reconocer y dirigir la mirada a un usuario que pase por las proximidades. Por tanto, no realizará ningún gesto. Sin embargo, cuando se encuentre en modo interacción, el robot, además de hacer seguimiento horizontal al usuario, ejecutará los gestos consignados por el secuenciador. Finalmente se encuentra el modo reposo en el que ambos subsistemas estarían inactivos.

Queda justificado, por tanto, el uso de un nivel superior en la arquitectura que controle ambos subsistemas.

\subsection{DESARROLLO}

Toda la implementación de la interfaz de acciones está concentrada en el nodo que recibe el mismo nombre (Figura 7).

El servidor de acciones toma como entrada un objetivo ordenado por el cliente (en nuestro caso el secuenciador), por ejemplo: "Modo interacción y expresión tristeza". Entonces, el servidor cambia su estado a "Procesando" y, mientras el cambio de modo y la realización del gesto se está llevando a cabo, el secuenciador irá recibiendo información sobre el estado de la orden. Esto último se hace mediante porcentaje, donde un $0 \%$ corresponde a "aún no iniciado" y, a medida que transcurre el tiempo, el porcentaje progresará hasta el $100 \%$ que significará que se ha cambiado el modo y el gesto ha sido ejecutado, si todo ha transcurrido correctamente. Si es así, el servidor cambia su estado a "liberado" y queda a la espera de recibir nuevas órdenes.

\section{RESULTADOS OBTENIDOS}

En esta sección se van a exponer los resultados obtenidos en los subsistemas desarrollados.

Para analizar el sistema de seguimiento con Kinect se muestra la evolución del error respecto al tiempo, así como la evolución de la referencia y el ángulo del servomotor.

Como se puede observar en la Figura 8, se han diseñado una serie de experimentos en forma de cambios en la posición del usuario bruscos (escalón) y progresivos (rampa).

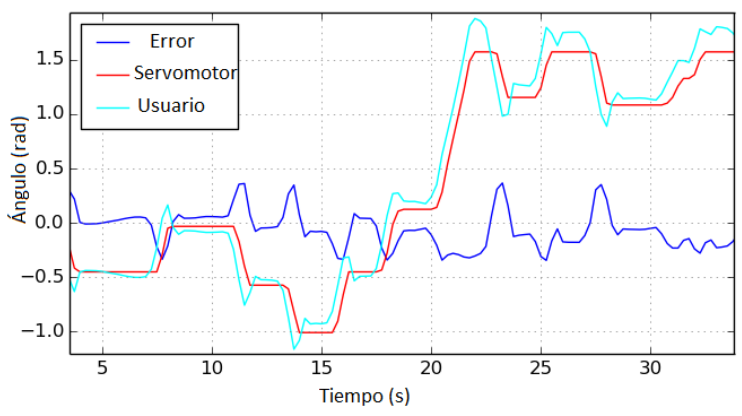

(a) Evolución temporal en el plano guiñada

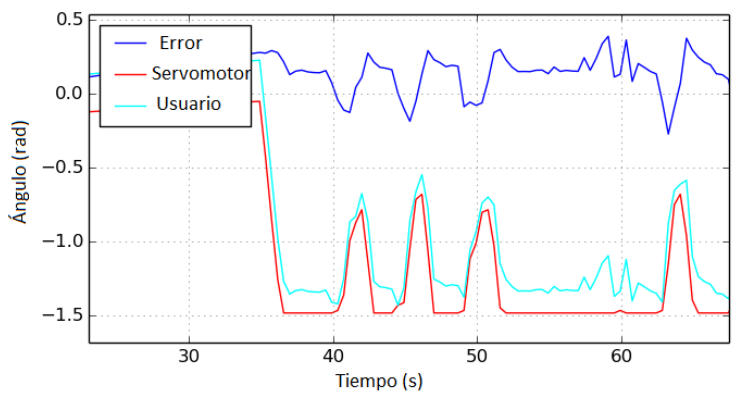

(b) Evolución temporal en el plano de cabeceo

Figura 8: Respuestas temporales del sistema de seguimiento

La respuesta de los servomotores es rápida y sobreamortiguada, con un retardo menor de $0.15 \mathrm{~s}$. Además, se puede apreciar que, aunque la referencia oscile en algunos casos, los esfuerzos de control no comienzan a ser apreciables hasta que no se produzcan cambios más significativos, de acuerdo al tratamiento no lineal que se le ha dado al error.

Por su parte, el error de posicionamiento se mantiene en una banda de $0 \pm 0.1 \mathrm{rad}$ en el estacionario. Dicha banda se produce porque para errores pequeños la 
salida de la función sigmoide es aproximadamente cero, por lo que no se excita a la señal de control.

Esto último es beneficioso, puesto que la cabeza del robot no está continuamente reacomodándose una vez está centrada en el usuario. De lo contrario, el servomotor se podría quedar oscilando en torno a la posición final y producir un efecto negativo en la interacción.

Para finalizar este apartado, se muestran los resultados obtenidos en el subsistema de expresión gestual (Tabla II).

Tabla 2: Expresión gestual de Sacarino II

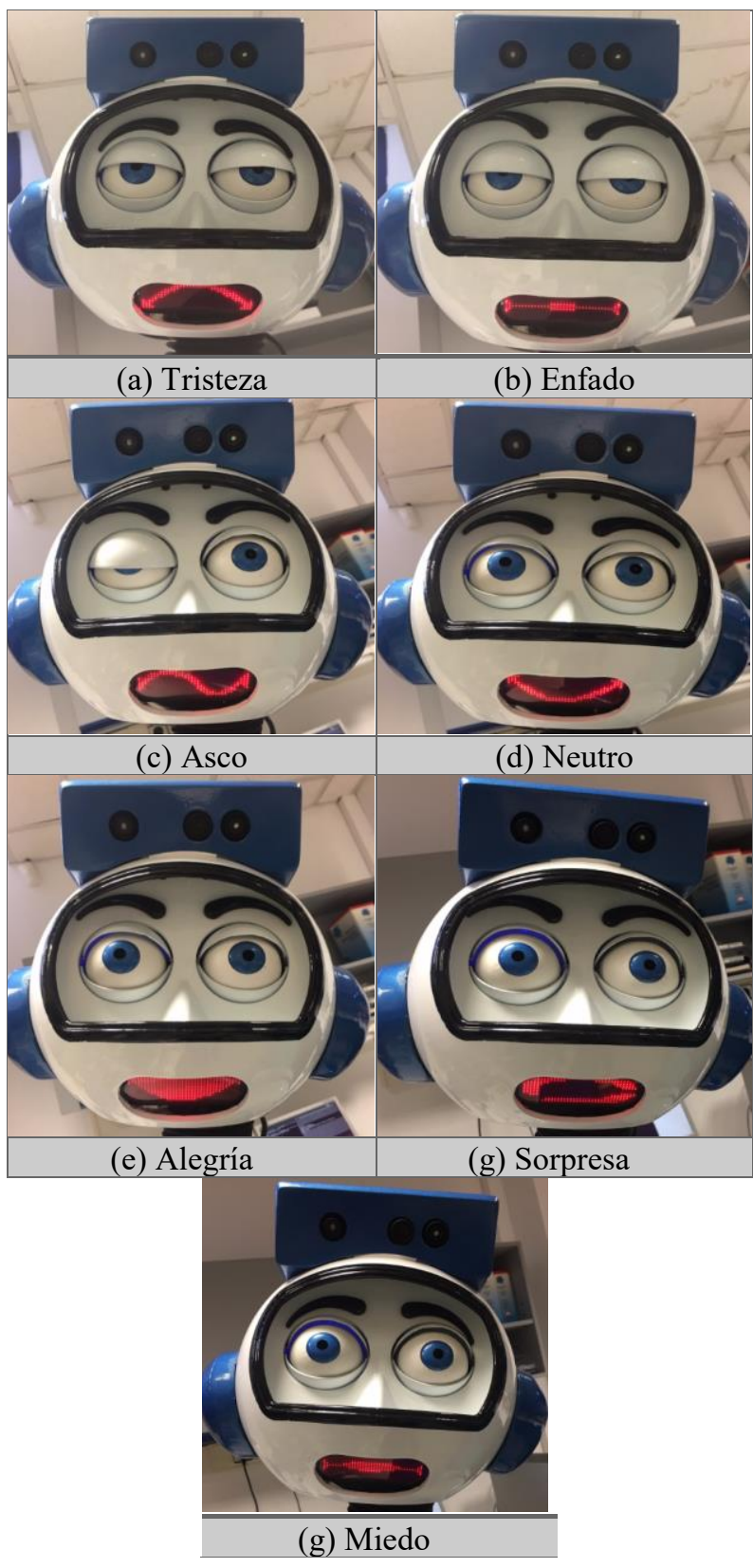
CONCLUSIONES

El objetivo fijado era el de dotar al robot Sacarino II de una mayor expresividad, para lograr un mejor impacto en la interacción hombre máquina.

Los estándares de comportamiento social dictan que es importante, a la hora de tratar con una persona, mirarla a los ojos, signo de estar activo en la conversación; y por otra parte, la gesticulación y expresividad facial acentúan el acercamiento y lo hacen más humano, reforzando positivamente la interacción. Para abordar este objetivo se ha propuesto un sistema de interacción basado en dos módulos.

Se ha desarrollado un primer módulo de detección de usuarios mediante un láser SICK. Una vez seleccionado uno de los usuarios se realiza su seguimiento visual mediante sensor Kinnect integrado en la propia cabeza. Este seguimiento realiza de forma suave evitando oscilaciones mediante la inclusión de un controlador no lineal sigmoidal.

Los experimentos han demostrado un comportamiento natural en el seguimiento de usuarios.

Por otra parte, el subsistema de expresión gestual basado en FACS, proporciona al robot una gran expresividad, y una experiencia que resulta agradable para el usuario.

Para coordinar estos dos subsistemas, la biblioteca actionlib de ROS se ha mostrado como una herramienta muy útil a la hora de proporcionar al secuenciador del robot una realimentación en tiempo real de las órdenes que éste envía al sistema de interacción.

A partir del desarrollo propuesto en este proyecto y como líneas futuras de desarrollo se propone por un lado, la posible incorporación de un sistema de detección de expresiones emocionales del usuario así como sistemas adicionales de interacción.

\section{Agradecimientos}

Este trabajo ha sido financiado por el Ministerio de Ciencia e Innovación, proyecto de investigación fundamental DPI2014-56500.

\section{Bibliografía}

[1] A. J. Sánchez, «Desarrollo de un sistema de visón-3D para su integración en un robot móvil social,» 2014 
[2] D. C. L. Matovelle, «Diseño y Construcción de una cabeza mecatrónica de aspecto realista,» 2013.

[3] Dautenhahn, K., Nehaniv, C. L. (1999). "Embodiment and interaction in socially intelligent lifelike agents. In Computation for Metaphors, Analogy and Agent", Ed., LNAI 1562, pp. 102-142.

Springer

[4] Eduardo Zalama Casanova; Jaime Gómez García-Bermejo; Samuel Marcos; Salvador Domínguez; Raúl Feliz; Roberto Pinillos. Sacarino, a service robot in a hotel environment.Advances in Intelligent Systems and Computing, 253, pp. 1 - 14. Madrid. Springer (USA). ISSN 2194-5357. (2013)

[5] Joaquin Lopez, Diego Perez, Eduardo Zalama and Jaime Gomez-Garcia-Bermejo. BellBot - A Hotel Assistant System Using Mobile Robots. International Journal of Advanced Robotic Systems, Vedran Kordic, Aleksandar Lazinica, Munir Merdan (Ed.) 2013. InTech. ISBN: 1729-8806. (2013

[6] P. Ekman and W. Friesen. Facial Action Coding System: A Technique for the Measurement of Facial Movement. Consulting Psychologists Press, Palo Alto, 1978.

[7] «Página oficial de ROS,» [En línea]. Available: http://wiki.ros.org/.

[8] R. Pinillos, «Diseño de un robot social y validación de servicios en entornos hoteleros,». Trabajo Fin de Master. Universidad de Valladolid. 2014.

[9] «Repositorio oficial de ROS,» [En línea]. Available: http://wiki.ros.org/leg_detector

[10] «Repositorio oficial de ROS,» [En línea]. Available: http://wiki.ros.org/openni_tracker

[11] Roberto Pinillos, Samuel Marcos, Raul Feliz, Eduardo Zalama, Jaime Gómez-GarcíaBermejo. «Long-Term Assessment of a Service Robot in a Hotel Environment». Robotics and Autonomous System. ISSN: 0921-8890. DOI: 10.1016/j.robot.2016.01.014 (2016).

[12] S. Moriello, «Robots sociales, la nueva generación.,» 2008. [En línea]. Available: http://www.tendencias21.net/Robots-sociales-lanueva-generacion_a2833.html. [Último acceso:
201705 19].

[13] SSC-32 Servo Controller Driver. Lynxmotion. http://www.lynxmotion.com/p-395-ssc-32servo-controller.aspx 